\title{
Generalized Joint Hypermobility among School- going children in Majmaah region, Saudi Arabia
}

\section{Mohamed Sherif Sirajudeen ( $\nabla$ m.sirajudeen@mu.edu.sa )}

Majmaah University College of Applied Medical Sciences https://orcid.org/0000-0002-6934-5352

\section{Mohamed Waly}

Majmaah University College of Applied Medical Sciences

\section{Mazen Alqahtani}

Majmaah University College of Applied Medical Sciences

\section{Msaad Alzhrani}

Majmaah University College of Applied Medical Sciences

Hariraja Muthusamy

Majmaah University College of Applied Medical Sciences

\section{Radhakrishnan Unnikrishnan}

Majmaah University College of Applied Medical Sciences

\section{Rashmi Saibannavar}

Majmaah University College of Applied Medical Sciences

\section{Wafa Alrubaia}

Majmaah University College of Applied Medical Sciences

\section{Anandh Vaiyapuri}

Majmaah University College of Applied Medical Sciences

\section{Gopal S Nambi}

Prince Sattam bin Abdulaziz University College of Applied Medical Sciences

\section{Research article}

Keywords: Beighton score, Joint hypermobility, Ligamentous laxity, Prevalence, Children, Saudi Arabia Posted Date: December 3rd, 2019

DOI: https://doi.org/10.21203/rs.2.17255/v2

License: (9) This work is licensed under a Creative Commons Attribution 4.0 International License. Read Full License 
The authors have withdrawn this preprint from Research Square 